\title{
Pancytopenia due to IKZF1 mutations
}

INSERM

\section{Source}

INSERM. (1999). Orphanet: an online rare disease and orphan drug data base.

Pancytopenia due to IKZF1 mutations. ORPHA:317473

Pancytopenia due to IKZF1 mutations is a rare syndrome with combined

immunodeficiency characterized by a variable clinical presentation ranging from

asymptomatic individuals to potentially life-threatening, recurrent bacterial infections

associated with progressive loss of serum immunoglobulins and B cells. 